\title{
MICROORGANISMOS PRESENTES EN FONENDOSCOPIOS, MANOS, CAVIDAD ORAL Y NASAL DE ESTUDIANTES DE UNA FACULTAD DE MEDICINA
}

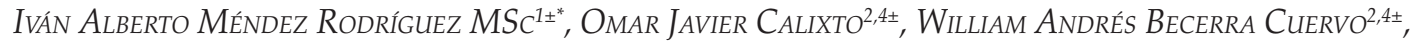 \\ Juan Felipe Vásquez ${ }^{2 \pm}$, Juan Sebastián Bravo Ojeda MD ${ }^{3}$, Diana Patricia Pachón Barinas MSc ${ }^{1 \pm}$. \\ ${ }^{1}$ Docente de la Facultad de Medicina de Universidad Militar Nueva Granada. \\ ${ }^{2}$ Estudiante de Medicina Universidad Militar Nueva Granada. \\ ${ }^{3}$ Médico, Servicio de Infectología Hospital Militar Central. \\ ${ }^{4}$ ACEMED-UMNG. \\ ${ }^{ \pm}$Integrante del grupo de patogenicidad microbiana de la Facultad de Medicina de la Universidad Militar Nueva Granada.
}

\begin{abstract}
Resumen
La formación académica de estudiantes de medicina implica interacción con pacientes y superficies del entorno hospitalario, los microorganismos presentes allí pueden ser adquiridos y transmitidos con los riesgos consecuentes para la comunidad hospitalaria. El presente es un estudio descriptivo cuantitativo donde se analizan muestras provenientes de estudiantes de medicina ( $\mathrm{n}=155)$ en rotaciones de 3 semanas, tomando hisopados de nariz, boca, manos y fonendoscopio, gram y cultivo en agar sangre, incubación 24 horas a $37^{\circ} \mathrm{C}$ y $5 \%$ de $\mathrm{CO}_{2}$. Se hizo la identificación mediante gram y pruebas de biotipificación convencionales y susceptibilidad antibiótica según técnica de kirby-bauer. Los hallazgos aquí presentados evidencian alta frecuencia de bacterias patógenas en las fuentes de aislamiento, siendo relevante la presencia del Staphylococcus aureus en un $41,7 \%$ y la resistencia a antibióticos tanto por gram positivos como por gram negativos; por tanto, es de considerar la implementación de estrategias que minimicen la circulación de estos microorganismos en el ambiente hospitalario. El objetivo de este estudio es establecer la frecuencia de los microorganismos aislados en estudiantes de una Facultad de Medicina.
\end{abstract}

Palabras clave: Estetoscopio, fonendoscopios, contaminación, higiene.

\section{MICROORGANISMS IN STETHOSCOPES, HANDS,ORAL AND NASAL CAVITY FROM STUDENTS IN A SCHOOL OF MEDICINE}

\begin{abstract}
The academic training of medical students involves interaction with patients, where microorganisms in contact with different surfaces can be acquired and transmitted with the consequent risk to the community hospital. This is a quantitative descriptive study on students of medicine $(n=155)$ with clinic rotations of 3 weeks, swabs were taking from the nose, mouth, hands and stethoscope, following by gram and blood agar cultures, incubation 24 hours at $37^{\circ} \mathrm{C}$ and $5 \% \mathrm{CO}_{2}$, final Identification was performed by gram, biotyping tests and antibiotic susceptibility by kirby-bauer technique.The findings presented here show high frequency of bacterial pathogens in source isolation, to be relevant the presence of Staphylococcus aureus in $41.7 \%$ and resistan cetoantibiotics both grampositive
\end{abstract}

* Correspondencia: Iván Alberto Méndez Rodríguez iván.mendez@unimilitar.edu.co. Dirección Correspondencia: Tr. 3 No 49-00. Facultad de Medicina, Universidad Militar Nueva Granada, Bogotá, Colombia. 
andgram-negative, therefore is to consider the implementation of strategies to minimize the movement of these microorganisms in the hospital environment. The objective of this study is to establish the frequency of microorganisms isolated from students at a medical school.

Key words: Stethoscope, contamination, hygiene.

\title{
MICRO-ORGANISMOS PRESENTES EM ESTETOSCÓPIOS, MÃOS, CAVIDADE ORAL E NASAL DE ESTUDANTES DE UMA FACULDADE DE MEDICINA
}

\begin{abstract}
Resumo
A formação acadêmica dos estudantes de medicina implica interação com pacientes e superfícies do entorno hospitalar, os micro-organismos presentes ali podem ser adquiridos e transmitidos com os riscos consequentes para a comunidade hospitalar. O presente é um estudo descritivo quantitativo onde foram analisadas amostras provenientes de estudantes de medicina $(n=155)$ em rotações de 3 semanas, colhidas do nariz, da boca, das mãos e de estetoscópios, gram e cultivo em ágar sangue, incubação 24 horas a $37^{\circ} \mathrm{C}$ e $5 \%$ de $\mathrm{CO}_{2}$, foi feita a identificação mediante gram e testes de biotipificação convencionais e suscetibilidade antibiótica segundo técnica de kirby-bauer. Os descobrimentos aqui apresentados evidenciam alta frequência de bactérias patógenas nas fontes de isolamento, sendo relevante a presença do Staphylococcus aureus em $41,7 \%$ e a resistência a antibióticos tanto por gram positivos como por gram negativos; portanto, é necessário considerar a implementação de estratégias que minimizem a circulação destes micro-organismos no ambiente hospitalar. O objetivo deste estudo foi estabelecer a frequência dos micro-organismos isolados em estudantes de uma Faculdade de Medicina.
\end{abstract}

Palavras chave: Estetoscópio, contaminação, higiene.

\section{Introducción}

El proceso de formación universitaria de los estudiantes de medicina contempla diferentes actividades que implican interacción con el ambiente hospitalario, desde el contacto con pacientes y familiares de estos, hasta procedimientos quirúrgicos y de diagnóstico.

Lo anterior se traduce en una alta circulación de microorganismos que facilitan la colonización del personal al cuidado de la salud y los pacientes, en especial aquellos con alteraciones inmunes y enfermedades crónicas, así mismo, la contaminación de superficies, elementos de uso médico y de diagnóstico.

Se estima que aproximadamente de 6-14\% de los pacientes que ingresan a un hospital pueden desarrollar una infección nosocomial. Así mismo, datos estadísticos indican en Estados Unidos, cerca de 90.000 pacientes mueren al año a causa de estas infecciones $(1,2)$.
El Staphylococcus aureus es un coco gram positivo, catalasa positivo, coagulasa positivo, capsulado, algunas de las cepas pueden ser toxigénicas; en la actualidad las infecciones se asocian a patrones de sensibilidad o resistencia a la oxacilina, denominándolas $S$. aureus meticilino sensible SAMS o resistente SAMR respectivamente. Igualmente, el perfil de elementos de virulencia encontrados el cassete SCC mec, la leucocidinapanton Valentine y la modulina sensible al fenol en las cepas adquiridas en comunidad (CA-MRSA del inglés community-associated-methicilin resistance Staphylococcus aureus) o adquiridas en centros del cuidado de la salud (HA-MRSA del inglés Health care adquired methicilin resistance staphylococcus aureus) (3).

Dentro de los Enterococcus spp, existen dos especies de significancia epidemiológica, E. faecalis y E. faecium, ambos habitantes del tracto gastrointestinal en humanos, patógenos oportunistas asociados a infecciones en sitio quirúrgico, vías urinarias y cuadros 
sépticos en el ámbito de las IACS. Estos cocos gram positivos catalasa negativo, son versátiles en cuanto a sus perfiles de resistencia a los antibióticos; la mayoría de las cepas son constitutivamente resistentes a las cefalosporinas y exhiben un patrón de resistencia a los glicopéptidos con cinco fenotipos conocidos (VanA-VanE) (4).

Tanto los Staphylococcus spp como los Enterococcus spp tienen buena tolerancia a las condiciones de desecación y carencia de nutrientes, por lo cual son importantes contaminantes de superficies.

Las cepas de Staphylococcus aureus meticilino resistente (SAMR), han emergido durante en las últimas décadas como el principal patógeno causante de infección asociada al cuidado de la salud (IACS).Así mismo, los Enterococcus spp resistentes a vancomicina se encuentra en aproximadamente un cuarto de todos los casos reportados, convirtiendo a estos dos microorganismos en los de más difícil control según la Sociedad Americana de Epidemiologia de Salud (SHEA - Society for Health care Epidemiology of America) $(2,5)$.

Se acepta que los microorganismos con algún perfil de resistencia a los antibióticos se transmiten eficientemente de persona a persona y/o fómites (2), siendo las superficies y el equipo médico fácilmente contaminados como el caso de Enterococcus spp. $(4,6)$ y $S$. aureus incluso a partir de material plástico $y$ telas de uso frecuente a nivel hospitalario (7).

Microorganismos aislados en trabajadores de la salud $y$ en implementos de uso diario (fonendoscopios y esfingomanómetros), han corroborado la afirmación que estos actúan como vehículo y reservorio de microorganismos potencialmente patógenos como el Staphylococcus aureus $(8,9,10,11)$; para el caso particular de los fonendoscopios, esto se asocia a una limpieza infrecuente $(12,13)$.

El aislamiento de cepas de Staphylococcus aureus junto a otros microorganismos es fundamental para la vigilancia al interior de cada centro asistencial. La presencia de $S$. aureus fue determinada en trabajadores de la salud (médicos generales, residentes, especialistas, internos y estudiantes de medicina) $(14,15)$, donde se evaluó el impacto en la detección y manejo de portadores asintomáticos evidenciando la disminución en la tasa de infección en una unidad de cuidados inten- sivos pediátricos (14). De igual forma se ha reportado resistencia uniforme a la oxacilina y eritromicina, un $52,9 \%$ a kanamicina y un $47 \%$ a gentamicina en $S$. aureus (10). Además, se ha identificado que solo el aseo constante puede disminuir la colonización por microorganismos patógenos a nivel de implementos como el fonendoscopio (16). El estricto cuidado que se debe tener en las barreras que prevengan la transmisión de patógenos en el entorno hospitalario, así como el uso racional de antibióticos evitando la selección de cepas con mayor perfil de resistencia (10).

En un intento por realizar una evaluación global de la resistencia antibiótica de microorganismos en diferentes entidades hospitalarias a nivel nacional, los informes epidemiológicos disponibles evidencian una baja sensibilidad del Staphylococcus aureus a la oxacilina (66\% en hospitales Universitarios).Se notificó además, Enterococcus spp resistente a vancomicina, así mismo, resistencia a cefalosporinas de tercera generación en cepas de Enterobacter spp y Klebsiella pneumoniae junto con un perfil de resistencia superior al 50\% para Acinetobacter spp a amikacina, aztreonam, ciprofloxacina y ceftazidima (17).

El reconocimiento de los trabajadores al cuidado de la salud y el personal en entrenamiento como vehículo-reservorio de agentes potencialmente patógenos, hace necesario mantener una estrecha vigilancia a la higiene personal y sus implementos como medida para disminuir las tasas de infección y resistencia antimicrobiana.

El presente estudio pretende identificarlos microorganismos y el patrón de resistencia antibiótica a partir de muestras nasales, orofaríngeas, manos y fonendoscopios de estudiantes de medicina durante sus rotaciones clínicas en un hospital universitario.

\section{Método y sujetos}

Estudio transversal, descriptivo cuantitativo. Los participantes fueron estudiantes del programa de medicina de la Facultad de Medicina de la Universidad Militar Nueva Granada que tuvieron prácticas clínicas en el Hospital Militar Central en el tiempo de duración del estudio (2009-2011). Se realizó un muestreo por conveniencia entre estudiantes de VI a XII semestre.

Se consideraron como criterios de inclusión: 1. Ser estudiante matriculado cursando entre sexto 
y duodécimo semestre de Medicina, 2. Ser rotante en área clínica en el Hospital Militar Central, durante dos semanas anteriores a la toma de la muestra, 3. Firma de consentimiento informado. Los criterios de exclusión usados durante el estudio fueron: 1 . Ingesta de antibiótico en el mes anterior a la toma de muestra con selectividad por la vía área superior, 2 . Estudiantes en tratamiento con inmunomoduladores durante el mes previo a la toma de muestra, 3. Sintomáticos respiratorios, definidos como persona que presenta tos y expectoración por más de 15 días.

Posterior a la firma del consentimiento informado, se tomó una muestra a cada estudiante de hisopado nasofaríngeo, orofaríngeo de manos y del diafragma y campana del fonendoscopio.

Las muestras, independientes de la fuente, fueron inicialmente teñidas con gram, sembradas por agotamiento en una placa de agar sangre (AS 5\% de glóbulos rojos de cordero), se realizó la lectura de las colonias que crecieron en agar sangre, verificando el tipo de hemólisis y la morfología mediante una segunda tinción de gram. Las bacterias con morfología correspondiente a bacilos gram negativos fueron cultivadas en agar Eosin Metilene Blue (EMB) para su identificación como fermentadores o no de la lactosa, posteriormente se procedió a realizar las pruebas bioquímicas: TSI (Triple sugariron), SIM (sulfuro, indol, motilidad), Citratos, Urea, MR-VP (Methil Red-Voges-proskauer).

A los cocos gram positivos alfa hemolíticos aislados de orofaringe, se les realizó la prueba de la catalasa y se indicaron como Streptococcus del grupo viridans si la prueba era negativa. A los cocos gram positivos alfa hemolíticos aislados de nasofaringe sugestivos de Streptococcus pneumoniae, se les realizó la prueba de la catalasa y la prueba de la optoquina en agar sangre. A los cocos gram positivos gama hemolíticos, se les efectuó la prueba de la catalasa y se sembraron en agar bilis esculina. A los cocos gram positivos beta hemolíticos, se procedió a realizar la prueba de catalasa, con un resultado positivo se sembró en agar salado manitol (ASM), con un disco de novobiocina, igualmente se les realizó la prueba de coagulasa en tubo; si la prueba de catalasa dio negativa, se sembró en AS con un disco de bacitracina. Respecto a los bacilos gram positivos aislados, se procedió a realizar las pruebas bioquímicas de fermentación de azúcares y motilidad.
De manera aleatorizada algunos aislamientos fueron confirmados empleando el kit de serotipificación Sthapytec plus (oxoid ${ }^{\circledR}$ ) para Staphylococcus aureus, el sistema de biotipificación CH50 para bacilos gram positivos y el sistema API20E para bacilos gram negativos.

En todos los casos las placas de agar y las pruebas en tubo se incubaron durante $24 \mathrm{~h}$ a $37^{\circ} \mathrm{C}$ en atmósfera de $5 \%$ de $\mathrm{CO}_{2}$.

Para identificar la susceptibilidad a los antibióticos se utilizó la técnica de Kirby-Bauer en medio MuellerHinton (MH); para cocos gram positivos se probaron los siguientes antibióticos: oxacilina, ampicilina sulbactam y cefalexina. Para bacilos gram negativos: ampicilina sulbactam, metronidazol, trimetoprimsulfametoxazol y gentamicina, para bacilos gram positivos: penicilina, ciprofloxacina, metronidazol, y clindamicina. Las placas se incubaron durante $24 \mathrm{~h}$, a $37^{\circ} \mathrm{C}$ en atmósfera de $5 \%$ de $\mathrm{CO}_{2}$.

Todos los procedimientos se realizaron de acuerdo con los lineamientos del Comité Nacional para Estándares de Laboratorio Clínico (NCCLS del inglés National Committee for Clinical Laboratory Standards), excepto en la determinación del patrón de resistencia a la meticilina para los Staphylococcus aureus, donde no se empleó el disco de cefoxitina $(30 \mu \mathrm{g})$ sino el de oxacilina $(1 \mu \mathrm{g})(18)$.

Los datos se registraron en una base de datos en Excel y se manejaron con el programa SPSS versión 15 para Windows (statistical package for social sciencies).

\section{Resultados}

La población estudiada correspondió a 100 mujeres $(64,5 \%)$ y 55 hombres $(35,5 \%)$. Las características demográficas de los participantes en el estudio se detallan en la tabla 1.

Algunas de las rotaciones, dentro de las especialidades médico quirúrgicas básicas, tuvieron la siguiente proporción en el muestreo: cardiología $4.5 \%$, cirugía cardiovascular 3.9\%, urología 3.2\%, anestesiología $3.2 \%$, salud pública $1.9 \%$, ortopedia $1.3 \%$, neuropediatría $1.3 \%$, neonatología $1.3 \%$, infectopediatría $1.3 \%$, consulta externa $1.3 \%$, medicina Infantil $1.3 \%$, dermatología $0.6 \%$, oftalmología $0.6 \%$, cirugía pediátrica $0.6 \%$ y neumología $0.6 \%$. 
Se obtuvieron 848 aislamientos a partir de 648 muestras. La distribución de aislamientos porcentual en cavidad nasal fue del $22,7 \%$, para cavidad oral del $19,7 \%$, en manos del $28,8 \%$ y en fonendoscopios del 20,2\%, con aislamientos negativos de 8,6\%, ver Figura 1.

Según su morfología la distribución encontrada fue de cocos gram positivos en un $73,4 \%$, bacilos gram negativos del $6,6 \%$ y bacilos gram positivos del 7,4\% no tipificable el $1,4 \%$ y sin crecimiento el $11,2 \%$, ver Tabla 2.
Las pruebas de biotipificación permitieron la identificación de los siguientes microorganismos: Staphylococcus aureus 41,7\%, Streptococcus grupo v Viridans 15,4\%, Staphylococcus coagulasa negativo - SCN 10,2\%, Streptococcus spp 7,8\%, Streptococcus Grupo A0,2\%, Enterococcus spp 0,4\%, Escherichia coli 2\%, Proteus spp 0,5\%, Yersinia spp 0,5\%, Klebsiella spp 0,9\%, Listeria spp $6,7 \%$, Eubacterium spp 1,9\%, y otros géneros en menos del $0,4 \%$.

Tabla 1. Características demográficas de la población del estudio.

\begin{tabular}{|c|l|c|c|c|c|c|c|}
\hline \multirow{2}{*}{ SEMESTRE } & \multicolumn{1}{|c|}{$\begin{array}{c}\text { ROTACIÓN } \\
\text { RESPECTIVA }\end{array}$} & \multicolumn{2}{|c|}{ HOMBRES } & \multicolumn{3}{c|}{ MUJERES } \\
\hline & & n & \% & N & $\%$ & n & \% \\
\hline VI & MEDICINA INTERNA & 8 & 14,5 & 29 & 29,0 & 37 & 23,9 \\
\hline VII & $\begin{array}{l}\text { CIRUGÍA GENERAL Y } \\
\text { SUBESPECIALIDADES }\end{array}$ & 7 & 12,7 & 20 & 20,0 & 27 & 17,4 \\
\hline VIII & PEDIATRÍA & 7 & 12,7 & 9 & 9,0 & 16 & 10,3 \\
\hline IX & GINECOLOGÍA Y OBS- & 6 & 10,9 & 9 & 9,0 & 15 & 9,7 \\
\hline TERICIA / UROLOGÍA & X & 12 & 21,8 & 14 & 14,0 & 26 & 16,8 \\
\hline internado & URGENCIAS & 15 & 27,3 & 19 & 19,0 & 34 & 21,9 \\
\hline Total & & 55 & 100 & 100 & 100 & 155 & 100 \\
\hline
\end{tabular}

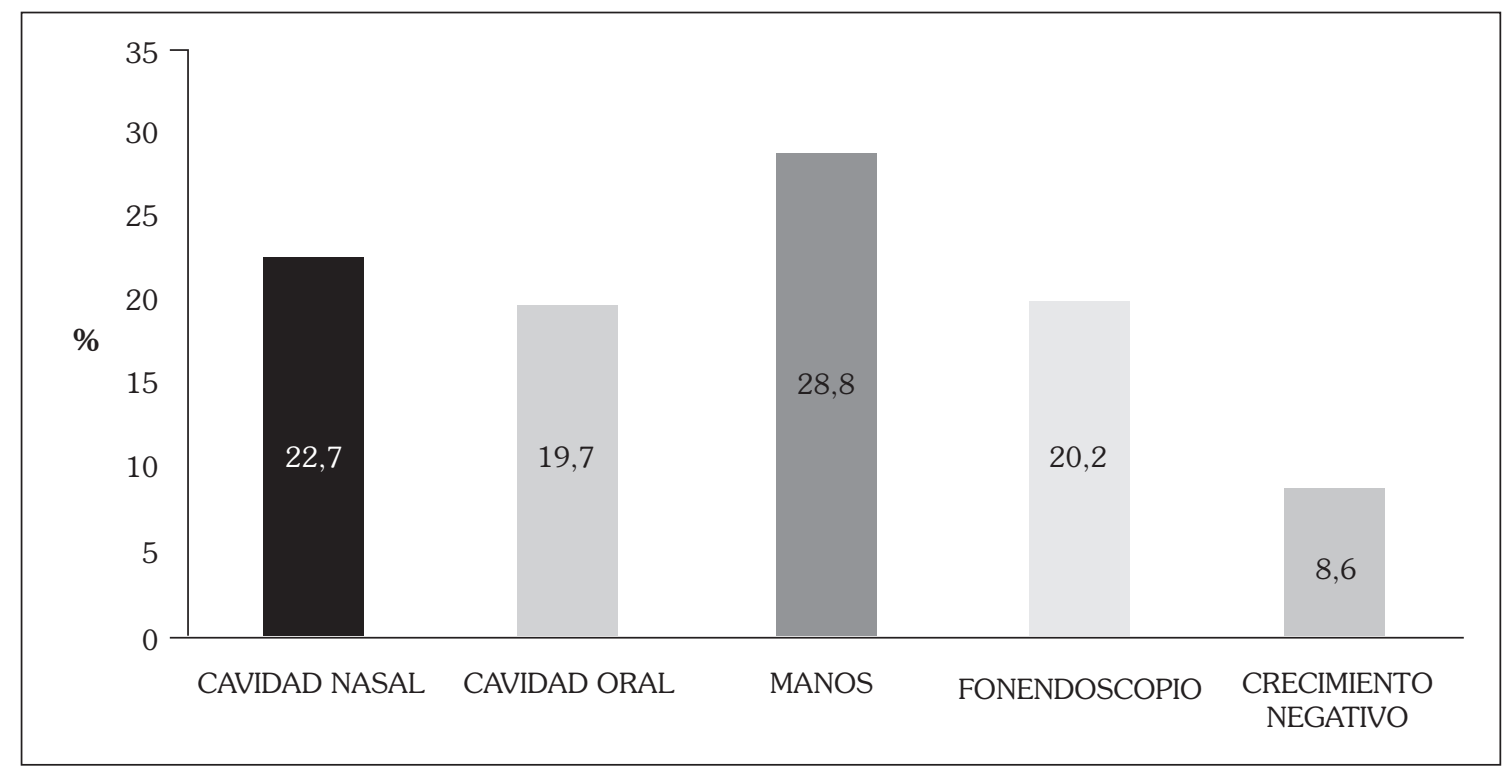

Figura 1. Distribución porcentual de aislamientos por sitio anatómico. 
Tabla 2. Distribución porcentual de aislamientos según morfología y tipificación.

\begin{tabular}{|c|c|c|c|c|c|}
\hline \multicolumn{6}{|c|}{ TOTAL: 620} \\
\hline \multicolumn{2}{|c|}{$\begin{array}{l}\text { COCOS GRAM POSITIVOS } \\
n=455(73.4 \%)\end{array}$} & \multicolumn{2}{|c|}{$\begin{array}{l}\text { BACILOS GRAM POSITIVOS } \\
n=46(7,42 \%)\end{array}$} & \multicolumn{2}{|c|}{$\begin{array}{l}\text { BACILOS GRAM NEGATIVOS } \\
n=41(6,61 \%)\end{array}$} \\
\hline MICROORGANISMO & n (\%) & MICROORGANISMO & n (\%) & MICROORGANISMO & n (\%) \\
\hline S.aureus & $308(67,7)$ & \multirow{7}{*}{\multicolumn{2}{|c|}{$\begin{array}{l}\text { Listeria spp } \\
\quad 6(100)\end{array}$}} & E.coli & $18(43,9)$ \\
\hline S.viridans & $104(22,85)$ & & & Klebsiella spp & $10(24,4)$ \\
\hline S.epidermidis & $28(6,15)$ & & & Yersinia spp & $4(9,75)$ \\
\hline S.saprophyticus & $1(0,21)$ & & & Proteus spp & $3(7,31)$ \\
\hline Enterococus spp & $3(0,65)$ & & & Enterobacter spp & $1(2,43)$ \\
\hline Streptococcus spp & $9(1,80)$ & & & Shewanella spp & $1(2,43)$ \\
\hline \multirow[t]{3}{*}{ S.pyogenes } & $2(0,43)$ & & & Aeromonas spp & $1(2,43)$ \\
\hline & & & & Serratia spp & $1(2,43)$ \\
\hline & & & & Burkholderia spp & $1(2,43)$ \\
\hline
\end{tabular}

Los resultados de los microorganismos presentes con mayor frecuencia, según género, semestre y sitio anatómico se muestran en la tabla 3 para hombres y tabla 4 para mujeres, mientras en la figura 2 se muestra el perfil de susceptibilidad a los antibióticos por grupo morfológico.

\section{Discusión}

Los hallazgos aquí presentados muestran a los cocos gram positivos presentes en las cuatro fuentes como los microorganismos de mayor aislamiento, destacándose en particular el Staphylococcus aureus y los $\mathrm{SCN}$. De acuerdo con los datos presentados en las Tablas 2,3 y 4 , se observa que $S$. aureus es el microorganismo que más aislamientos positivos tuvo en los distintos sitios de aislamiento independientemente del semestre o rotación del estudiante. Se presentaron dos aislamientos de Streptococcus beta hemolítico del grupo A (S. pyogenes) en el grupo de internos.

Pocos estudios se han conducido evaluando personal de salud como portadores nasales, en orofarínge y en manos. En 2005, un estudio en personal hospitalario buscando la presencia de $S$. aureus, encontró nueve cepas, dos de los cuales fueron SAMR (19).

Es de resaltar que junto a la presencia de cocos gram positivos, se aislaron en manos y fonendoscopios en estudiantes de séptimo, octavo y noveno semestre bacilos gram negativos como E. coli, Klebsiella spp, Yersinia spp y entre los bacilos gram positivos aislados el más frecuente fue la Listeria spp.
Por ser biota normal se aislaron principalmente cocos alfa hemolíticos del grupo viridans en cavidad oral y Micrococcus spp en manos. No se aislaron cepas de Streptococcus pneumoniae, Pseudomonas aeruginosa o Stenotrophomonas spp.

Algunos de los microorganismos encontrados en las fuentes de aislamiento se caracterizan por ser patógenos oportunistas, en los aislamientos de manos se determinó la presencia de E. coli, Klebsiella spp, Enterobacter spp, Acinetobacter spp y Burkholderia spp, probablemente de diferentes orígenes entre ellos materia fecal y/o zonas húmedas hospitalarias.

En los fonendoscopios se aislaron cepas de Enterobacter spp, Klebsiella spp, Proteu spp, E. coli, Aeromonas spp, Serratia o Citrobacter spp y fue el único sitio donde se identificó la presencia de Enterococcus spp, mostrando la importancia del cuidado dado al fonendoscopio durante la práctica médica, siendo la materia fecal la principal fuente de contaminación con estas enterobacterias.

Nuestros hallazgos destacan mayor proporción de bacilos gram positivos $(7,4 \%)$ sobre los bacilos gram negativos $(6,6 \%)$ presentes en manos y estetoscopios, similar al reporte de Zuliani (15\% de bacilos gram positivos contaminantes de estetoscopios, con una tasa de 4 de 300 aislamientos para bacilos gram negativos) (8), así mismo, estudios previos revelan más de un $50 \%$ de contaminación por bacilos gram positivos en estetoscopios y otoscopios (20). 
Tabla 3. Microorganismos aislados según género, semestre, sitio anatómico y prevalencia.

\begin{tabular}{|c|c|c|c|c|c|c|c|c|c|c|c|c|}
\hline \multicolumn{13}{|c|}{ HOMBRES n (55) 35,48 \% } \\
\hline \multirow[t]{2}{*}{ SEMESTRE } & \multicolumn{3}{|c|}{ CAVIDAD NASAL } & \multicolumn{3}{|c|}{ CAVIDAD ORAL } & \multicolumn{3}{|c|}{ MANOS } & \multicolumn{3}{|c|}{ FONENDOSCOPIO } \\
\hline & $\begin{array}{c}\text { MICRO- } \\
\text { ORGANISMO }\end{array}$ & $\mathbf{n}$ & $\%$ & $\begin{array}{c}\text { MICRO- } \\
\text { ORGANISMO }\end{array}$ & $\mathbf{n}$ & $\%$ & $\begin{array}{c}\text { MICRO- } \\
\text { ORGANISMO }\end{array}$ & $\mathbf{n}$ & $\%$ & $\begin{array}{c}\text { MICRO- } \\
\text { ORGANISMO }\end{array}$ & n & $\%$ \\
\hline \multirow{3}{*}{$\begin{array}{c}\text { VI } \\
H: n=8\end{array}$} & S. aureus & 7 & 87,5 & S. aureus & 4 & 50 & S. aureus & 5 & 62,5 & S. aureus & 5 & 62,5 \\
\hline & S. viridans & 2 & 25 & S. viridans & 5 & 62,5 & S. viridans & 1 & 12,5 & Klebsiella spp & 1 & 12,5 \\
\hline & S. epidermidis & 2 & 25 & & & & S. epidermidis & 4 & 50 & E. coli & 1 & 12,5 \\
\hline \multirow{3}{*}{$\begin{array}{c}\text { VII } \\
\mathrm{H}: \mathrm{n}=7\end{array}$} & S. aureus & 5 & 71,4 & S. viridans & 6 & 85,7 & S.aureus & 7 & 100,0 & S. aureus & 7 & 100,0 \\
\hline & Listeria spp & 1 & 14,3 & S. aureus & 2 & 28,6 & Listeria spp & 3 & 42,9 & Listeria spp & 2 & 28,6 \\
\hline & Yersinia spp & 1 & 14,3 & E. coli & 2 & 28,6 & E. coli & 1 & 14,3 & S. epidermidis & 1 & 14,3 \\
\hline \multirow{3}{*}{$\begin{array}{c}\text { VIII } \\
\mathrm{H}: \mathrm{n}=7\end{array}$} & S. aureus & 5 & 71,4 & S.viridans & 4 & 57,1 & S. aureus & 4 & 57,1 & S. aureus & 4 & 57,1 \\
\hline & S. epidermidis & 1 & 14,3 & Proteus spp & 1 & 14,3 & Listeria spp & 2 & 28,6 & S. epidermidis & 1 & 14,3 \\
\hline & & & & E. coli & 1 & 14,3 & Klebsiella spp & 1 & 14,3 & & & \\
\hline \multirow{3}{*}{$\begin{array}{c}\text { IX } \\
H: ~ \\
n=6\end{array}$} & S.aureus & 4 & 66,7 & S.aureus & 3 & 50,0 & S. aureus & 6 & 100,0 & S. aureus & 4 & 66,7 \\
\hline & Streptococcus spp & 2 & 33,3 & S.viridans & 3 & 50,0 & Listeria spp & 1 & 16,7 & Klebsiella spp & 2 & 33,3 \\
\hline & & 0 & 0 & Klebsiella spp & 1 & 16,7 & E. coli & 1 & 16,7 & E. coli & 2 & 33,3 \\
\hline \multirow{3}{*}{$\begin{array}{c}X \\
H: n=12\end{array}$} & S. aureus & 6 & 50,0 & S.viridans & 10 & 83,3 & S. aureus & 12 & 100,0 & S. aureus & 6 & 50,0 \\
\hline & Listeria spp & 1 & 8,3 & E. coli & 2 & 16,7 & Listeria spp & 2 & 16,7 & Listeria spp & 3 & 25,0 \\
\hline & S. epidermidis & 1 & 8,3 & S. epidermidis & 1 & 8,3 & S. epideridis & 1 & 8,3 & $\begin{array}{l}\text { Serratia o } \\
\text { Citrobacter }\end{array}$ & 1 & 8,3 \\
\hline \multirow{3}{*}{$\begin{array}{c}\text { INTERNA- } \\
\text { DOS (XI-XII) } \\
\text { H: } n=15\end{array}$} & S. aureus & 11 & 73,3 & S. viridans & 12 & 90,0 & S. aureus & 15 & 100,0 & S. aureus & 8 & 53.3 \\
\hline & S. pyogenes & 1 & 6,7 & S. pyogenes & 1 & 6,7 & S. epidermidis & 2 & 13,3 & S. viridans & 1 & 6,7 \\
\hline & & & & & & & Listeria spp & 1 & 6,7 & S. viridans & 1 & 6,7 \\
\hline
\end{tabular}

Tabla 4. Microorganismos aislados según género, semestre, sitio anatómico y prevalencia.

\begin{tabular}{|c|c|c|c|c|c|c|c|c|c|c|c|c|}
\hline \multicolumn{13}{|c|}{ MUJERES n (100) $64.52 \%$} \\
\hline \multirow[t]{2}{*}{ SEMESTRE } & \multicolumn{3}{|c|}{ CAVIDAD NASAL } & \multicolumn{3}{|c|}{ CAVIDAD ORAL } & \multicolumn{3}{|c|}{ MANOS } & \multicolumn{3}{|c|}{ FONENDOSCOPIO } \\
\hline & $\begin{array}{c}\text { MICRO- } \\
\text { ORGANISMO }\end{array}$ & $\mathbf{n}$ & $\%$ & $\begin{array}{c}\text { MICRO- } \\
\text { ORGANISMO }\end{array}$ & $\mathbf{N}$ & $\%$ & $\begin{array}{c}\text { MICRO- } \\
\text { ORGANISMO }\end{array}$ & $\mathbf{n}$ & $\%$ & $\begin{array}{c}\text { MICRO- } \\
\text { ORGANISMO }\end{array}$ & $\mathbf{n}$ & $\%$ \\
\hline \multirow{3}{*}{$\begin{array}{c}\text { VI } \\
M: n=29\end{array}$} & S. aureus & 12 & 41,4 & S. aureus & 12 & 41,4 & S. aureus & 20 & 69,0 & S. aureus & 15 & 51,7 \\
\hline & Proteus spp & 1 & 3,4 & S. viridans & 16 & 55,2 & Klebsiella spp & 1 & 3,4 & Listeria spp & 8 & 27,6 \\
\hline & Listeria spp & 2 & 6,9 & S. saprophyticus & 1 & 3,4 & E. coli & 1 & 3,4 & Enterobacter spp & 1 & 3,4 \\
\hline \multirow{3}{*}{$\begin{array}{c}\text { VII } \\
M: n=20\end{array}$} & S. aureus & 12 & 60,0 & S. viridans & 19 & 95,5 & S. aureus & 19 , & 95,0 & Proteus spp & 1 & 5,0 \\
\hline & Klebsiella spp & 1 & 5,0 & S. aureus & 2 & 10,0 & Listeria spp & 3 & 15,0 & Enterococcus spp & 1 & 5,0 \\
\hline & Yersina spp & 1 & 5,0 & E. coli & 1 & 5,0 & $\begin{array}{c}\text { Acinecto } \\
\text { bacter spp }\end{array}$ & 1 & 5,0 & E. coli & 1 & 5,0 \\
\hline \multirow{3}{*}{$\begin{array}{c}\text { VIII } \\
\text { M: } n=9\end{array}$} & S. aureus & 6 & 66,7 & S. viridans & 7 & 77,8 & S. aureus & 7 & 77,8 & S. aureus & 5 & 55,6 \\
\hline & Yersinia spp & 2 & 22,2 & Listeria spp & 2 & 22,2 & Listeria spp & 1 & 11,1 & Listeria spp & 1 & 11,1 \\
\hline & Listeria spp & 1 & 11,1 & E. coli & 1 & 11,1 & & & & Klebsiella spp & 1 & 11,1 \\
\hline
\end{tabular}




\begin{tabular}{|c|c|c|c|c|c|c|c|c|c|c|c|c|}
\hline \multicolumn{13}{|c|}{ MUJERES n (100) $64.52 \%$} \\
\hline \multirow[t]{2}{*}{ SEMESTRE } & \multicolumn{3}{|c|}{ CAVIDAD NASAL } & \multicolumn{3}{|c|}{ CAVIDAD ORAL } & \multicolumn{3}{|c|}{ MANOS } & \multicolumn{3}{|c|}{ FONENDOSCOPIO } \\
\hline & $\begin{array}{c}\text { MICRO- } \\
\text { ORGANISMO }\end{array}$ & $\mathbf{n}$ & $\%$ & $\begin{array}{c}\text { MICRO- } \\
\text { ORGANISMO }\end{array}$ & $\mathbf{N}$ & $\%$ & $\begin{array}{c}\text { MICRO- } \\
\text { ORGANISMO }\end{array}$ & $\mathbf{n}$ & $\%$ & $\begin{array}{c}\text { MICRO- } \\
\text { ORGANISMO }\end{array}$ & $\mathbf{n}$ & $\%$ \\
\hline \multirow{3}{*}{$\begin{array}{c}\text { IX } \\
M: n=9\end{array}$} & S. aureus & 9 & 100,0 & S. aureus & 6 & 66,7 & S. aureus & 4 & 44,4 & S. aureus & 1 & 11.1 \\
\hline & S. viridans & 1 & 11,1 & S. epidermidis & 1 & 11,1 & S. epidermidis & 111,1 & & Lissteriasspp & 1 & 7,1 \\
\hline & & & & E. coli & 1 & 7,1 & Shewanellaspp & 1 & 11,1 & E. coli & 1 & 7,1 \\
\hline \multirow{3}{*}{$\begin{array}{c}X \\
M: n=14\end{array}$} & S. aureus & 5 & 35,7 & S. viridans & 12 & 85,7 & S. aureus & 12 & 85,7 & Enterococcusspp & 2 & 14,3 \\
\hline & S. epidermidis & 5 & 35,7 & S. aureus & 1 & 7,1 & Listeriaspp & 2 & 14,3 & Listeriaspp & 1 & 7,1 \\
\hline & Streptococcusspp & 7 & 50,0 & E. coli & 1 & 7,1 & E. coli & 1 & 7,1 & Aeromonasspp & 1 & 7,1 \\
\hline \multirow{3}{*}{$\begin{array}{c}\text { INTERNADO } \\
\text { (XI - XII) } \\
\text { M: } \mathrm{n}=19\end{array}$} & S.aureus & 17 & 89,5 & S. viridans & 16 & 84,42 & Listeriaspp & 3 & 15,8 & S. aureus & 8 & 42,1 \\
\hline & S.viridans & 1 & 5,3 & S. aureus & 5 & 26,3 & Klebsiellaspp & 1 & 5,3 & S. epidermidis & 5 & 26,3 \\
\hline & S.epidermidis & 1 & 5,3 & & & & Burkholderiaspp & 1 & 5,3 & Listeriaspp & 5 & 26,3 \\
\hline
\end{tabular}

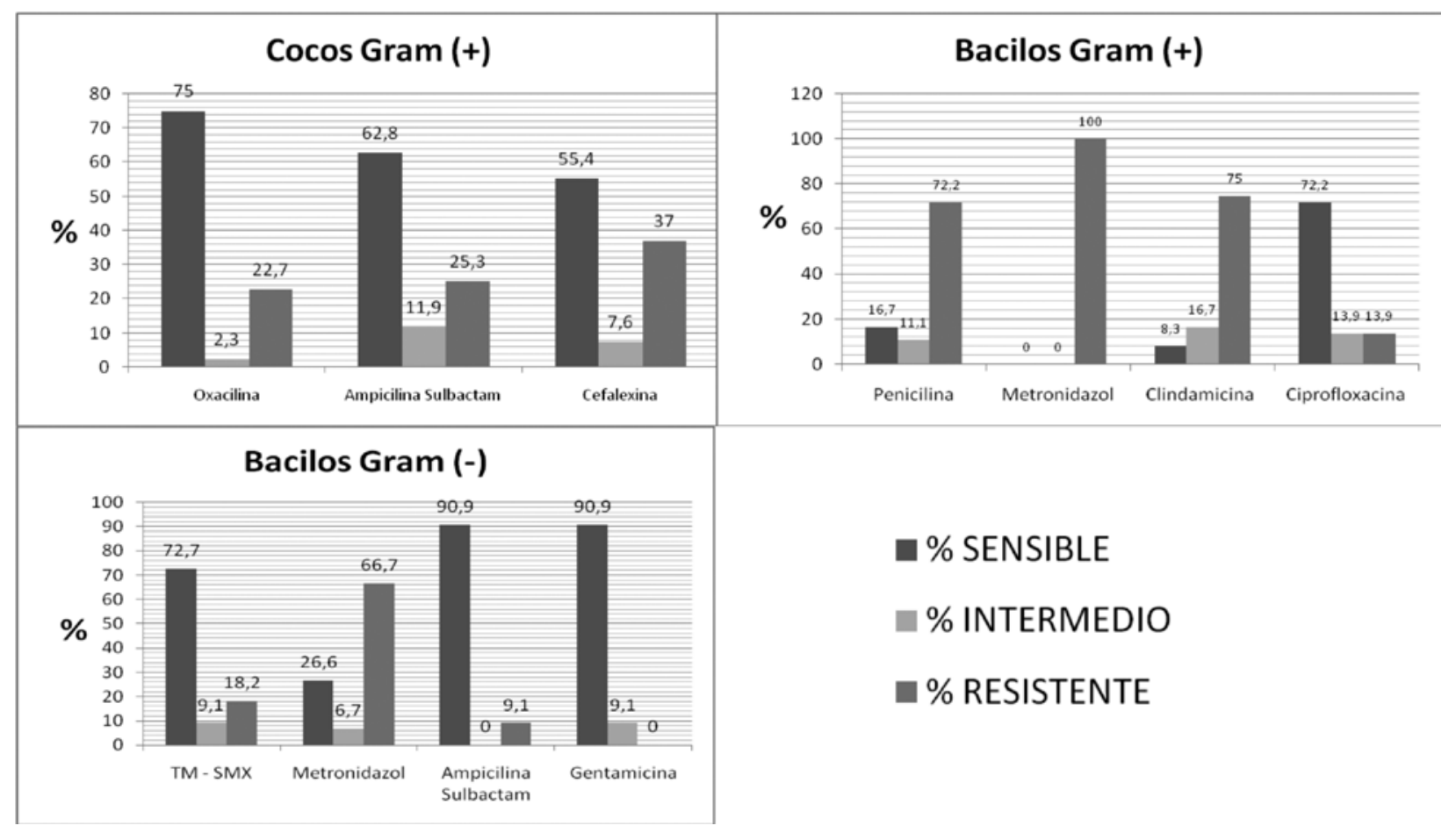

Figura 2. Perfil de susceptibilidad a los antibióticos (TMP-SXT: trimetoprimsulfametoxazol). 
En los cuatro sitios de aislamiento se encontró una alta frecuencia de Listeria spp, por las características de supervivencia en fómites, es de importancia el proceso de desinfección de superficies físicas y corporales para el control de la carga de este microorganismo.

Entre los gram negativos se aislaron cepas de E. coli, Klebsiella spp, Yersinia spp, Enterobacter spp, Proteus spp., la mayoría con sensibilidad a trimetoprim sulfametoxazol, ampicilina-sulbactam y gentamicina, siendo importante la resistencia al metronidazol; no se obtuvieron aislamientos de Pseudomonas aeruginosa, donde estudios previos revelan una tasa entre el 1\% al 10\% en estetoscopios $(8,9,21)$.

La presencia de la mayoría de gram positivos y gram negativos en manos y estetoscopios, sugiere la adquisición por inadecuados hábitos e higiene o durante el examen físico al paciente, aspecto que indica que no se realiza el adecuado lavado de manos y asepsia del estetoscopio después de auscultado un paciente. Se han realizado múltiples estudios evaluando la efectividad de la limpieza del estetoscopio sobre la contaminación microbiana encontrando una sensible disminución de hasta el 96,3\% para microorganismos portadores de resistencia como los SAMR (20, 21, 22, 23).

El perfil de susceptibilidad a los antibióticos usados para los cocos gram positivos, mostró un significativo $22 \%$ de resistencia a la oxacilina (figura 2), mayor a lo reportado en el estudio clásico de Cohen a finales de los noventas en estetoscopios (7,3\%) y otoscopios $(9,5 \%)(9)$ y menor a lo informado en servicios de emergencia en un hospital en Nueva Jersey (32\%) (24).

El estudio de Shobha demostró que cepas del Staphylococcus spp aisladas de cavidad nasal y estetoscopios, estas poseían niveles de resistencia cercanos al 70\% para ampicilina, amoxicilina/clavulanato y TMP/SXT y sensibilidad a vancomicina, ciprofloxacina y amikacina. Álvarez y su grupo, encontraron resistencia en más del $47 \%$ a oxacilina, eritromicina, kanamicina y gentamicina en las cepas evaluadas de S. aureus $(10,19)$.

Desde el 2006, el NCCLS, recomienda en su guía el uso del disco de cefoxitina para la detección de resistencia a los beta lactámicos mediada por el gen meca. Sin embargo, en el 2008 un estudio contemplando 1611 cepas de Staphylococcus aureus donde se comparo el uso del disco de oxacilina $1 \mu \mathrm{g}$ y de cefoxitina $30 \mu \mathrm{g}$, indicó que entre el 1,5\% a 4,4\% de falsos sensibles se presentan cuando se emplea el disco de oxacilina (25). En el presente trabajo se encontró un $75 \%$ de cepas sensibles a la oxacilina, tomando en cuenta lo anterior, la sensibilidad estaría en el orden del 71\% para los hallazgos de este estudio.

Se aislaron dos cepas de Streptococcus pyogenes sensibles a oxacilina, ampicilina sulbactam y cefalexina, correspondientes a internos con fuente de aislamiento nasal y oral, esto es importante reconociendo la posibilidad que sean portadores asintomáticos y la recomendación de manejo profiláctico para erradicar el microorganismo.

Los datos generales del antibiograma para cepas de cocos gram positivos, evidencian niveles de resistencia entre $22 \%$ a $37 \%$ a oxacilina, ampicilina-sulbactam y cefalexina mientras en los bacilos gram positivos la resistencia está entre $72 \%$ a $100 \%$ a penicilina, metronidazol, clindamicina, siendo la más baja a la ciprofloxacina (14\%). Para los bacilos gram negativos la resistencia fue de un $67 \%$ a metronidazol y al trimetoprimsulfametoxazol(TMP/SXT) y ampicilinasulbactam menor al 20\%, teniendo susceptibilidad $100 \%$ a gentamicina.

Este estudio no evaluó la sensibilidad de los Staphylococcus aureus a la vancomicina, aunque se conoce una muy baja prevalencia de cepas con resistencia a este antibiótico y que los métodos de identificación de cepas con perfil intermedio o resistente se deben mediante microdilución en caldo, screening en agar con vancomicina o E-test.

Con base en los resultados de este trabajo, nuestro grupo de investigación estableció una prevalencia del 47,6\% (78/164) para el S. aureus a partir de cavidad nasal y manos de estudiantes de la Facultad de Medicina $(n=82)$ entre diciembre de 2011 y febrero de 2012, con una distribución del 34,6\% de cepas SAMR (por cefoxitina $30 \mathrm{mg}$ ) y un $6,4 \%$ de cepas con resistencia intermedia-VISA a la vancomicina (por E-test).

\section{Conclusiones}

El reconocimiento de los trabajadores y el personal de salud en entrenamiento como vehículo o reservorio de agentes patógenos, hace necesario mantener una 
estrecha vigilancia de las labores de higiene en el personal sanitario y sus implementos como medida para disminuir las tasas de infección hospitalaria.

Estudios han referido que los estetoscopios y elementos de laboratorio que actúan como fómites, permiten la supervivencia de $S$. aureus al menos durante dos semanas; igualmente, un estudio que evaluó los cobertores de tela para fonendoscopios, evidenció que también son elementos sujetos de contaminación tanto por cocos gram positivos y en menor proporción por bacilos gram positivos y bacilos gram negativos $(26,27)$.

El estudio de Hill y colaboradores del 2006 en el Reino Unido, reporto una baja tasa de limpieza a los fonendoscopios en personal médico y tan solo con recomendaciones y seguimiento a la limpieza se demostró una disminución de la colonización especialmente de $S$. aureus(28).

En otro estudio realizado a los fonendoscopios de Médicos en Michigan, Estados Unidos, el 48\% realizaba limpieza de éste de manera semanal, el 37\% de mensual, el 7\% anual y un 7\% nunca habían realizado limpieza alguna a este instrumento (29).

De igual forma el uso de cubiertas de fonendoscopios también se asocia con mayor colonización bacteriana (30); por lo cual, el uso de alcohol diariamente para la limpieza de equipo tiene un impacto significativo en la disminución de la contaminación de esos instrumentos hasta de un $25 \%(23,31)$.

Nuestro grupo evaluó estudiantes de medicina entre diciembre del 2011 a febrero del 2012, como posibles portadores del Staphylococccus aureus, estableciendo un 47,6\% de aislamientos positivos, con una distribución del 34,6\% de cepas oxacilina resistenteSAMR (determinado por discos de cefoxitina $30 \mu \mathrm{g}$ oxacilina $1 \mu \mathrm{g}$ ) y $6,4 \%$ con resistencia intermedia a la

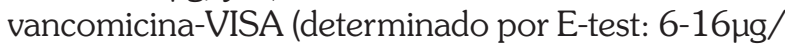
$\mathrm{ml})$, datos sin publicar.

De este estudio no se puede concluir que los estudiantes de medicina pudieran ser exclusivamente los transmisores de microorganismos de importancia a nivel hospitalario. Pero si es indicativo que desde la formación médica se deben promover conductas adecuadas de higiene personal y del cuidado de los elementos de trabajo en su práctica diaria, así como la realización de tamizajes periódicos a los estudiantes con el fin de detectar microorganismos con potencial patógeno y poder realizar una intervención que limite la circulación de los mismos.

Finalmente, será de interés futuro relacionar los hallazgos microbiológicos con la incidencia de infección hospitalaria asociada a estos microorganismos durante el tiempo de rotación de los estudiantes en cada servicio.

\section{Agradecimientos}

A los estudiantes de la Facultad de Medicina de la Universidad Militar, Bogotá, Colombia. Al Doctor Daniel Toledo por su apoyo en el manejo estadístico de los datos. A la Doctora Clara Benavides por su colaboración en la elaboración del cronograma de obtención de muestras y al Fondo de Investigaciones de la Universidad Militar Nueva Granada subvención MED402.

\section{Referencias bibliográficas}

1. González J. Infección Nosocomial por Acinetobacter baumanii multirresistente en unidades de cuidados críticos. Enfermeríalntegr. 2005; 72: 15-22.

2. Diekema D, Dogson K, Sigurdardottir B, Pfaller M. Rapid Detection of Antimicrobial - Resistant Organism Carriage: an Unmet Clinical Need. J ClinMicrobiol. 2004;42: 2879-2883.

3. David M,Daum R. Community-Associated Methicillin-Resistant Staphylococcus aureus: Epidemiology and Clinical Consequences of an Emerging Epidemic. ClinMicrobiol Rev. 2010; 23(3): 616-687

4. Cetinkaya Y, Falk P, MayhallCG.Vancomycin-resistant enterococci. Clin Microbiol Rev. 2000; 13(4): 686-707.

5. Hernández I, Toraño G, González M, Gonzales L. Staphylococcus aureus resistente a la meticilina: detección de portadores entre niños hospitalizados y niños sanos de la comunidad. Rev Cub Med Trop. 2003; 55: 153-61.

6. Mundy LM, Sahm DF, Gilmore M. Relationships between enterococcal virulence and antimicrobial resistance. ClinMicrobiol Rev. 2000;13(4):513-22

7. Neely AN, Maley MP. Survival of enterococci and staphylococci on hospital fabrics and plastic.J Clin Microbiol. 2000; 38(2): 724-726

8. Zuliani ME, Maldonado AF, Bercial ME, Pedroso SA. Stethoscope: a friend or an enemy?.Rev Paul Med. 2002; 120: 13-15.

9. Nuñez S, Moreno A, Green K, Villar J. The stethoscope in the emergency department: a vector of infection. Epidemiol Infect. 2000; 124: 233-237.

10. Álvarez E, Velazco E, Nieves B, Vivas G, Gutierrez B. Detección de portadores de Staphylococcus aureus resistente a la meticilina en una unidad de alto riesgo neonatal. RevFac Farmacia. 2005; 47: 16-20.

11. Villamil AS, Rodriguez C, Badia MB, Lopez-Moral LS, Zilberman JM, Salinas RL, et al. Los manguitos del esfigmomanómetro son reservorios de bacterias potencialmente patógenas. Rev Argent Cardiol. 2004; 72: 9-13.

12. Nuñez S, Moreno A, Rodriguez I, Garcia P, Hernandez JR, Izquierdo $\mathrm{C}$. El estetoscopio como vector de la infección nosocomial en urgencias. Emergencias. 1999;11:281-285. 
13. Mitchell A, Dealwis N, Collins J, Chew K, Taylor R, Schwab U, Narayanan M.Stethoscope or 'Staphoscope'? Infection by auscultation.J Hosp Infect. 2010; 76(3): 278-279.

14. Mendoza CN, Barrientos CM, Panizza VF, Concha BR, Romero PP, Barhona MF, et al. Prevencion de la infección intrahospitalaria por Sthaphylococcus aureus resistente a meticilina mediante el manejo de portadores. Rev Chil Infect. 2000; 17(2): 129-134.

15. Youngster I, Berkovitch M, Heyman E, Lazarovitch Z, Goldman M.The stethoscope as a vector of infectious diseases in the paediatric division.ActaPaediatr. 2008; 97(9): 1253-1255.

16. Whittington AM, Whitlow G, Hewson D, Thomas C, Brett SJ. Bacterial contamination of stethoscopes on the intensive care unit.Anaesthesia. 2009; 64(6): 620-624.

17. Robledo C, Robledo J. Panorama de la resistencia a los antibióticos en Colombia. En: Salvatierra R, Benguigui Y. Resistencia antimicrobiana en las Américas: magnitud del problema y su contención. Washington. Organización Panamericana de la Salud; 2000. p.141.

18. Wikler MA, Conckreill FR, Craig WA, Dudley MN, Eliopoulos GM, Hecht DW, et al. Performance standards for antimicrobial disk susceptibility tests. National Committee for Clinical Laboratory Standards. Approved standard. National Committee for Clinical Laboratory Standards.19th edition, 2009.

19. Shobha KL, Rao PS, Thomas J. Survey of Staphylococcus isolates among hospital personnel, environment and their antibiogram with special emphasis on methicillin resistance. Ind J Med Microbiol.2005; 23: 186-188.

20. Cohen HA, J, Matalon A, Mayan R, Beni S, Barzilai A. Stethoscopes and otoscopes - a potential vector of infection? FamPract. 1997; 14: 446-449.

21. Schroeder A, Schroeder MA, D'Arnico F. What's growing on your stethoscope? (and what you can do about it). J FamPract. 2009; 58: 404-409.
22. Thofern UAR. Bacterial contamination of hospital physicians stethoscopes. Letters to editor.Infect control HospEpidemiol. 2000; 21: 558-559.

23. Parmar R, Valvi C, Sira P, Kamat J. A prospective, randomized, double bind study of comparative efficacy of immediate versus daily cleaning of stethoscope using $66 \%$ ethyl alcohol. Ind $J$ Med Sci. 2004; 58: 423-429.

24. Merlin MA, Wong ML, Pryor PW, Rynn K, Marques-Baptista A, Perritt R, et al. Prevalence of methicillin-resistant Staphylococcus aureus on the stethoscopes of emergency medical services providers. PrehospEmergCare. 2009; 13: 71-74.

25. Williams CH, Davis DL.Methicillin-resistant Staphylococcus aureus fomite survival. Clin Lab Sci. 2009; 22: 34-38.

26. Millan MW, Hall M, Pringle T, Buchanan K. Bacterial contamination of fabric stethoscope coverts: The velveteen rabbit of health care?Infect Control Hosp Epidemiol. 2001; 22: 653-655.

27. Broekema N, Van T, Monson T, Marshall S, Warshauer D. Comparison of Cefoxitin and Oxacillin Disk Diffusion Methods for Detection of mecA-Mediated Resistance in Staphylococcus aureus in a Large-Scale Study. J ClinMicrobiol. 2009; 47(1): 217-219.

28. Hill C, King T, Day R. A strategy to reduce MRSA colonization of stethoscopes. J Hosp Infect. 2006; 62(1): 122-3.

29. Jones JS, Hoerle D, Riekse R. Stethoscopes: a potential vector of infection? Ann Emerg Med 1995; 26:296-9.

30. Wood MW, Lund RC, Stevenson KB. Bacterial contamination of stethoscopes with antimicrobial diaphragm covers. Am J Infect Control. 2007; 35(4): 263-6.

31. Bandi S, Uddin L, Milward K, Aliyu S, Makwana N. How clean are our stethoscopes and do we need to clean them? J Infect. 2008; 57(4): 355-6. 